\title{
Segmentation of low contrast-to-noise ratio images applied to functional imaging using adaptive region growing
}

\author{
Cabello J. ${ }^{a}$, Bailey A. ${ }^{b}$, Kitchen I. ${ }^{b}$, Guy M. ${ }^{c}$ and Wells K. ${ }^{a}$ \\ ${ }^{a}$ Centre for Vision, Speech and Signal Processing, Faculty of Engineering and Physical \\ Sciences, University of Surrey, Guildford, Surrey, GU2 7XH, UK; \\ ${ }^{b}$ Faculty of Health and Medical Sciences, University of Surrey, Guildford, Surrey, GU2 7XH, \\ UK; \\ ${ }^{c}$ Department of Nuclear Medicine, Royal Surrey County Hospital, Guildford, Surrey, GU2 \\ $7 \mathrm{XX}, \mathrm{UK}$
}

\begin{abstract}
Segmentation in medical imaging plays a critical role easing the delineation of key anatomical functional structures in all the imaging modalities. However, many segmentation approaches are optimized with the assumption of high contrast, and then fail when segmenting poor contrast to noise objects. The number of approaches published in the literature falls dramatically when functional imaging is the aim. In this paper a feature extraction based approach, based on region growing, is presented as a segmentation technique suitable for poor quality (low Contrast to Noise Ratio CNR) images, as often found in functional images derived from Autoradiography. The region growing combines some modifications from the typical region growing method, to make the algorithm more robust and more reliable. Finally the algorithm is validated using synthetic images and biological imagery.
\end{abstract}

Keywords: Segmentation, Region Grower, Functional Imaging, Autoradiography

\section{INTRODUCTION}

Image segmentation is one of the traditional branches of image analysis playing a key role in computer-aided diagnosis and therapy planning. These methods are represented by a multiplicity of different approaches. ${ }^{1}$ Conventional segmentation approaches are based on histogram thresholding, edge detection (active contours), watershed transformation and region extraction, or a combination of these.

Thresholding techniques are often discarded in medical imaging because these only consider histogram information, ignoring spatial information, and because of susceptibility to noise.

Edge detection based approaches have demonstrated good performance with high contrast images such as those found in Computed Tomography (CT) or Magnetic Resonance (MR), where boundaries are generally well defined, but for modalities with low contrast to noise ratio (CNR), as is often the case in functional imaging, boundaries appear diffuse and the level of noise is higher than that compared to CT and MR, due to low spatial resolution, significant partial volume effects and noise resulting from the statistical nature of radioactive decay. ${ }^{2}$

As a result, in 4D data, functional boundaries may be defined using temporal voxel characteristics. ${ }^{3-5}$ From the resulting time-curve activity (TAC) followed by some clustering method, such as K-means or MAPMRF, the segmentation is undertaken. Fuzzy C-means ${ }^{6}$ and Fuzzy Hidden Markov Chains ${ }^{2}$ have been other recent approaches used in PET.

As region-based methods, region growing is the most widely used technique for biomedical image segmentation due to its capability of handling noisy images and because it combines spatial connectivity and

Further author information: (Send correspondence to J. Cabello)

J. Cabello : E-mail: j.cabello@surrey.ac.uk, Telephone: +44 (0)1483 689856

Medical Imaging 2009: Image Processing, edited by Josien P. W. Pluim, Benoit M. Dawant,

Proc. of SPIE Vol. 7259, $725940 \cdot$ (c) 2009 SPIE

CCC code: $1605-7422 / 09 / \$ 18 \cdot$ doi: $10.1117 / 12.811325$

Proc. of SPIE Vol. 7259 725940-1 
intensity information. This technique assumes that neighboring pixels within the same region have some similarity criterion, most often based on intensity values.

Some alternative approaches have also been presented in the literature..$^{7-11}$ Two measures such as the gradient and the contrast were presented in Hojjatoleslami and Kittler. ${ }^{7}$ These discontinuity measures have demonstrated good performance with mammogram image data, ${ }^{12} \mathrm{CT}$ and MR images ${ }^{7}$ but are too sensitive to noise when segmenting autoradiograms.

The typical homogeneity criterion, where a candidate pixel is appended to the growing region if its intensity value is within some user-defined margins, is used in multiple approaches published in the literature. ${ }^{8-11}$ These user-defined margins are computed in an initial learning step, previous to the proper image segmentation, in Pohle and Toennies. ${ }^{8}$ Noticeably $\mathrm{Ti}$ and $\mathrm{Ra}^{9}$ used competitive region growing by growing several seed regions at the same time, assuming that all the seeds are in direct contact. In Lu et al. ${ }^{10}$ every voxel is considered a seed at the beginning. Then the homogeneity criterion used to grow regions is based on the Pearson correlation coefficient between the time series of the voxel and the averaged time series of the voxels in a premerged region, and then compared to a pre-defined fixed threshold. In the end the resultant regions are the largest segmented regions. The homogeneity criterion is dynamically modified in Mancas et al. ${ }^{11}$ as the region grower evolves. A Bayesian approach is used by $\mathrm{Pan}$ and $\mathrm{Lu}^{13}$ to model the bimodal histogram generated when a region grower operates between two different tissues, using fuzzy c-means to estimate the parameters that describe each distribution. However, this relies on reasonably good CNR.

In this paper we address the segmentation issue of low CNR images (as often found in functional biomedical image data) using a region based approach. An automatic region grower which tries to detect significant variations in the statistics in the region under segmentation, or significant increments of the region size being segmented is demonstrated using simulated and biological imagery, demonstrating the suitability of this algorithm to low CNR images.

\section{METHODOLOGY}

Classically, segmentation is defined as the partitioning of an image $I$ into $n$ non-overlapping regions $R_{d}$ as described by (1), which are homogeneous with respect to some characteristic. Some segmentation approaches assume a priori knowledge of the $n$ subregions. In the case of this algorithm $n$ is unknown.

$$
I=\cup_{d=1}^{n} R_{d}
$$

where $R_{k} \cap R_{j}=\emptyset$ for $k \neq j$, and each $R_{k}$ is connected.

The methodology now described is used for recursively segmenting objects represented in a low CNR image resulting in a fully labelled scene, without knowing the number of subsets a priori, $n$, in contrast with other approaches. This involves first simple removal of an assumed background component, followed by initialization and execution of the region grower, finally halting using some termination criteria. Once initial regions have been grown, then these are discarded from the original image, and the region grower is then recursively executed repeatedly until all pixels have been assigned to a segmented region.

It has been observed that after the autoradiogram has been segmented, there still exist some regions with low levels of uptake (low intensity values) that have not been properly segmented. Therefore, once the region grower has segmented all the possible regions, the whole process is applied to an intensity-inverted version of the same autoradiogram. The result of undertaking this second run provides a final image with more regions properly segmented, easing the overall analysis of the autoradiograms.

\subsection{Pre-processing}

Prior to applying a region grower on the autoradiograms, the raw image data is first pre-processed. This pre-processing is comprised of two steps: First a thresholding step, where the background is removed, and secondly a filtering step, where an anisotropic filter is applied to the autoradiogram, to reduce statistical noise of the autoradiogram that may produce over-segmentation of certain regions. These two steps are described below. 


\subsubsection{Thresholding}

Initially images are thresholded before being segmented to remove the background noise. This is undertaken by computing the histogram of the image, and assuming this comprises of a set of $m$ Gaussian-distributed components, all the pixels belonging to the first Gaussian distribution of the histogram are then set to zero.

\subsubsection{Anisotropic Filtering}

Given the high statistical noise that functional data, such as autoradiographic data, usually exhibits, an anisotropic filter is first applied to the autoradiograms. An anisotropic filter is a common method to enhance the image quality in computer graphics, first introduced by Perona and Malik. ${ }^{14}$ It is anisotropic because it is space variant, i.e. it is not homogeneous in all directions. It smooths homogeneous regions and enhance sharp edges. An example of this can be found in Pan and Lu. ${ }^{13}$

\subsection{Region Grower}

Image segmentation algorithms based on region growing are always comprised of an initial step, where one or more seed pixels are defined. Once a seed pixel is placed, those pixels spatially connected to the seed pixel are considered as candidates to be joined to this seed pixel. This consideration is based on one ore more similarity criteria. These two steps as used in this approach, are detailed in the next two subsections.

\subsubsection{Initialization}

Most region growing techniques depend highly on the selection of the location of the initial seed pixels. In this work two assumptions are made in the initialization stage to automatically locate the seed pixels:

- The size of the minimum region to be segmented is $N \mathrm{x} N$ pixels. By making this assumption, we impose the smallest functional structure to be segmented as $2 N \mathrm{x} 2 N$ or more pixels, i.e. the smallest functional structure to be segmented is twice larger ( $2 N \mathrm{x} 2 N$ pixels) than the initial seed region $(N \mathrm{x} N$ pixels).

- The minimum distance between the segmented regions and a new seed pixel is $M$ pixels, where $M$ is set typically to be greater than $N$. This mitigates the effect of growing a new region which is actually part of an already segmented structure, that has been under-segmented.

The first seed region is centred on a randomly located pixel that occupies the highest intensity value in the image. This is then enclosed by a $N \times N$ seed region, $N$ being dependant on the size of the image. Some statistics, comprised of the mean, mode and standard deviation, and the highest pixel value in the seed region are calculated. For the specific case of the autoradiograms used in this work a region of 9x9 pixels was used (smallest functional structure is assumed to be $18 \times 18$ pixels).

\subsubsection{Dynamic Similarity Criterion}

A region grower is based on the similarity criterion, a criterion that candidate pixels have to fulfil to be appended to the region. Once the localized seed region statistics are computed, then a one pixel wide border, defined by the binary template $T$ (2), is considered, and each pixel of the border is evaluated.

$$
T=\left\{x_{i j} \notin \bigcup_{d=1}^{n} R_{d}: N\left(x_{i j}\right) \bigcap_{d=1}^{n} R_{d} \neq \emptyset\right\}
$$

where $N\left(x_{i j}\right)$ is the set of immediate neighbours of pixel $x_{i j} .{ }^{10}$ The considered pixel, $x_{i j}$, will be appended to the region $\left\{R_{d}\right\}$ if it fulfils the homogeneity criterion, i.e. if it is within the margins defined by (3):

$$
x_{i j} \begin{cases}\in R_{d} & \text { if } m_{o}-k \sigma_{1, o}<\hat{x}_{i j}<m_{o}+k \sigma_{2, o} \\ \notin R_{d} & \text { otherwise }\end{cases}
$$


where $\hat{x}_{i j}$ is the mean of the $3 \times 3$ region centred in $x_{i j}, m_{o}$ is the mode of the seed region, $\sigma_{1, o}$ is the standard deviation of the pixels in the seed region below $m_{o}, \sigma_{2, o}$ is the standard deviation of the pixels in the seed region above $m_{o}$ and $k$ is a parameter that controls the marginal step size. This parameter $k$ gives this region growing the capability of adapting the homogeneity criterion to the different difficulties exhibited in typical autoradiographic data, such as blurred edges and high statistical noise. An initial value of $k$ is set, but this is later increased dynamically ${ }^{11}$ (see Figure 1).

The use of two different standard deviations is based on the typical case where the histogram of the original seed region is modelled with a two component Gaussian mixture ${ }^{8}$ thus the mode of the histogram approximately sets the middle of the mixture, one standard deviation $\left(\sigma_{1, o}\right)$ represents the lower values of the mixture with intensity below $m_{o}$, in an approximate way, the variation of width of an assumed Gaussian like (but contaminated) distribution, and the second $\left(\sigma_{2, o}\right)$ is a similar descriptor for the higher values of the mixture. If the seed region is placed on a very homogeneous region and the histogram can be modelled with a single Gaussian, $\sigma_{1, o}$ and $\sigma_{2, o}$ will exhibit very similar values. This does not represent a problem for the correct modeling of the statistics of the seed region.

Some parameters are measured from the intensity histogram of the region at each iteration $t$ to be used by the termination criterion based on the intensity histogram (explained below):

- mode of the current region being segmented $\left(m_{t}\right)$.

- the difference between the mode intensity of the region at the current iteration $t\left(m_{t}\right)$ and the mode of the original seed region $\left(m_{o}\right)$, denoted as $\Delta_{m 1}$.

- the difference of the mode intensity of the region at iteration $t\left(m_{t}\right)$ and the previous mode at iteration $t-1\left(m_{t-1}\right)$, denoted as $\Delta_{m 2}$.

Everytime the contour $T(2)$ is extracted, all the pixels contained in $T_{t}$ ( $t$ denoting the current iteration) are candidates to be appended to the region $R_{d, t}$. After appending those pixels that fulfil (3) a new region is obtained $R_{d, t+1}$. Using the same parameter $k$, the contour $T_{t+1}$ is extracted again in the next iteration $(t+1)$ from the new region $R_{d, t+1}$, to append those pixels that still fulfil (3).

Several iterations of the region grower will occur, using the initial parameter setting in (3), until no further pixels are appended to the region. Parameter $k(3)$ is then adaptively changed by a certain step size $(k=k+\triangle k)$ in response to the CNR of the image; the step size $\triangle k$ used in the experiments shown here has a value of one. By dynamically updating $k$, this parameter relaxes the conditions described in (3) so more pixels can be appended to the region being grown. Figure 1 shows the evolution of the boundary for automatically updated values of $k$, from the initial $k=3$ to the final $k=17$ for this example.

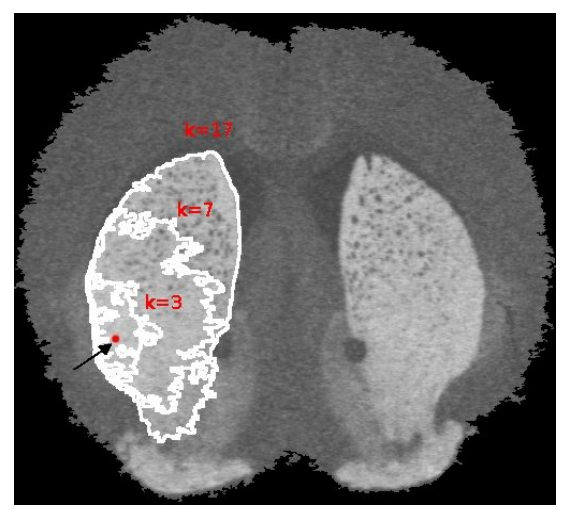

Figure 1. Evolution of the boundary as $k$ is automatically increased, relaxing the conditions described in (3), for $k=3$, $k=7$ and $k=17$. The initial seed region is indicated with an arrow. 
It is worth to make the difference at this point of the two different indices mentioned so far. One of them, $t$, is when using the same parameter settings defined by (3) (parameter $k$ is fixed), every time a new region $R_{d, t}$ is obtained, its contour $T_{d, t}$ is extracted to study its candidates. When no more pixels are appended to the region with the current configuration (3) parameter $k$ is updated, being the next iteration defined as $k+1$.

Everytime $k$ is updated there are a few tasks that are undertaken to determine if the region grower stopped appending pixels because it actually found an edge, or because, due to statistical noise or inhomogeneity of the region, the region grower found a false edge.

1. First the number of pixels appended to the region at each $k$ value is calculated. This function subsequently ensures the region grower does not over-grow. This is futher explained in Section 2.3.2 below.

2. Secondly, the number of iterations $t$ used for the current $k$ is stored. The physical explanation of this being that if $k$ is updated (increased $\triangle \mathrm{k}$ ), and subsequently few iterations $t$ occur with the new $k$ value until it halts again, is because those pixels being appended to the region exhibit very different intensity values compared to those already included in the segmented region. This is indicative of a possible change of texture, i.e. an edge.

3. Thirdly, the intensity value of those pixels contained in the contour of the region at the current iteration $T_{d, t}$, are penalized by a certain value. The reason for this penalization is because sometimes the region grower stops appending pixels due to statistical noise present in the region being segmented. By penalizing those pixels in the contour, if the intensity value of these pixels is close enough to those pixels in the region being segmented (it stopped due to the presence of statistical noise), although they are penalized they will be appended to the region grower anyway. On the other hand, if the intensity value of these pixels is far from those pixels in the region being segmented, the penalization will increase such difference of intensity value, avoiding these pixels to be appended.

$$
T_{d, t}=T_{d, t}-w\left(\mu_{s}-\mu_{c}\right)
$$

where $T_{d, t}$ is the outer contour of the current region $R_{d, t}, w$ is a weighting factor and $\mu_{s}$ and $\mu_{c}$ are the mean intensity value of $R_{d, t}$ and $T_{d, t}$ respectively. Note that $\mu_{s}$ and $\mu_{c}$ will always fulfil $\mu_{s}>\mu_{c}$, i.e. the pixel values of the region being segmented $R_{d, t}$ will always be higher than those in the contour $T_{d, t}$.

The weighting factor $w$ is obtained from the intensity distribution of those pixels contained in $R_{d, t}$ and $T_{d, t}$, being $p_{R}$ and $p_{T}$ their intensity distributions respectively. These intensity distributions, $p_{R}$ and $p_{T}$, will sometimes be very low populated, i.e. constructed with very few samples, when the region being considered is small. Therefore some considerations have to be taken to reduce the effect of statistical noise and produce undesirable results.

First $p_{R}$ and $p_{T}$ are modelled as a finite mixture model with the set of parameters $N\left(m, \sigma_{u}, \sigma_{l}\right)$ for each distribution, being $m$ the mode, and $\sigma_{u}$ and $\sigma_{l}$ the standard deviation of those pixels below and above $m$ respectively, similarly to that described above. Two sets of parameters are then obtained, $N_{R}\left(m_{R}, \sigma_{R, u}, \sigma_{R, l}\right)$ and $N_{T}\left(m_{T}, \sigma_{T, u}, \sigma_{T, l}\right)$.

Secondly a measure of the overlapping of $p_{R}$ and $p_{T}$ is computed. In order to do this two parameters are obtained, $p_{p_{R} \cap p_{T}}=p_{R} \cap p_{T}$, representing the measure of overlapping between both distributions, and $p_{p_{R} \cup p_{T}}=p_{R} \cup p_{T}$, used to normalize the overlapping between both distributions $p_{p_{R} \cap p_{T}}$. The overlapping measure $O$ is then defined by (5), being $O=[0,1)$. If $O=0$ means that there exist no overlapping between $p_{R}$ and $p_{T}$ (completely different tissues). The situation $O=1$ never arises as this would imply both distributions are identical, a situation which is impossible because at this point the region grower is stopped because the intensity pixel of those pixels in the contour can not be appended to the region grower due to the intensity disparity. 


$$
O=\frac{\sum p_{p_{R} \cap p_{T}}}{\sum p_{p_{R} \cup p_{T}}}
$$

After the model of each distribution, $N_{R}\left(m_{R}, \sigma_{R, u}, \sigma_{R, l}\right)$ and $N_{T}\left(m_{T}, \sigma_{T, u}, \sigma_{T, l}\right)$, and the overlapping $O$ have been computed, $w$ is then obtained following (6) to solve (4):

$$
w= \begin{cases}0 & \text { if } \sigma_{T, l}>2 \sigma_{T, u} \& \sigma_{R, u}>2 \sigma_{R, l} \\ 1 & \text { if } O=0 \quad\left(p_{R} \cap p_{T}=\emptyset\right) ; \\ 1-O & \text { otherwise } \quad\left(p_{R} \cap p_{T}>1\right) .\end{cases}
$$

\subsection{Termination Criteria}

Termination criteria are those conditions that have to be fulfilled to halt the region grower. These criteria have been built based on the empirical observation of the properties observed in digitized autoradiograms. These termination criteria are based on the points enumerated below:

- Change in intensity histogram of the region under segmentation.

- Difference between region sizes each time the parameter $k$ is updated.

- Edge detection based on image gradient.

\subsubsection{Termination criterion based on change in intensity histogram}

One of the conditions to stop the region grower is based on analysing the histogram of the segmented region at each iteration $t$. When segmenting low CNR imagery, we observe that the object being segmented is described by a Gaussian-like distribution, but as the region grower appends more pixels to the region, a second Gaussian distribution emerges initially as a long tail. At a certain point the histogram of the region will develop into a bimodal but frequently asymmetric Gaussian mixture (see Figure 2).

As the histogram of the region grown develops into a bimodal histogram, the difference between the mode intensity at iteration $t$ and the mode intensity computed from the initial seed region, defined by (7), is going to be significantly increased.

$$
\Delta_{m 1}=m_{t}-m_{o}
$$

where $m_{t}$ is the mode of the histogram at the iteration $t$ and $m_{o}$ is the mode computed from the original seed region.

In some cases the mixture distribution can be highly asymmetric, making it difficult to distinguish these two components (see Figure 3). This effect might happen also when the histogram results in a Landau-like distribution. One way of detecting which situation has occurred is by studying the evolution of the mode of the histogram at each iteration, defined by (8).

$$
\Delta_{m 2}=m_{t}-m_{t-1}
$$

where $m_{t}$ is the mode of the histogram at the current iteration and $m_{t-1}$ is the mode of the histogram at the previous iteration.

If the evolution of the histogram from the region being segmented results in a bimodal histogram, $\Delta_{m 1}$ and $\Delta_{m 2}$ will increase significantly at the iteration when the mode of the second distribution is higher than that of the first original distribution (indicated in an idealized situation in Figure 2). In this case the region being grown has flooded across different tissues. A hard threshold (shown in Figure 2) is applied here as the lowest (non zero) histogram bin between the two distributions. In this scenario a portion of the object 


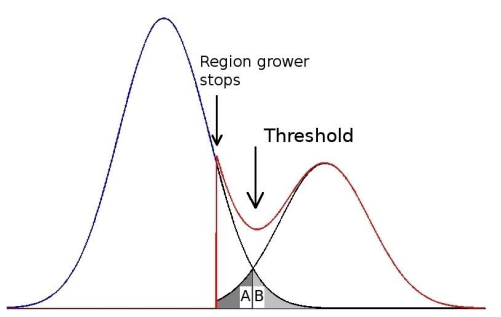

Figure 2. Idealized example of segmenting two different tissues resulting in a clearly distinguished bimodal distribution.

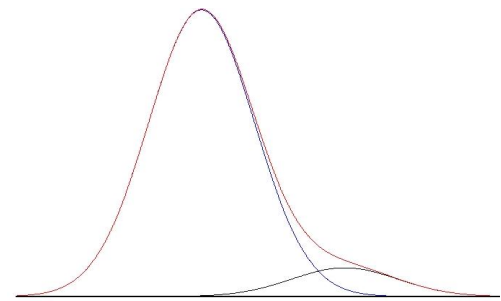

Figure 3. Idealized example of segmenting two different tissues with a highly asymmetric mixture distribution, making component separation difficult.

being segmented is incorrectly discarded (area A in Figure 2), and a portion of the segmented component is incorrectly assigned to the grown region (area B in Figure 2). After binarizing the segmented region, it is observed that pixels corresponding to area B, are not spatially connected to the grown region being segmented. These pixels are therefore easily removed using binary morphological operations.

In the case, where a highly asymmetric long tailed Gaussian histogram shape is observed (Figure 3 ), $\Delta_{m} 2$ will not exhibit the step change seen in the previous case. Therefore region growing may also be halted by detecting any potential over-segmentation from observing the change in region size as well, as described in Section 2.3.2.

In practice the intensity histogram of the region being segmented is not smooth, due to a mixture of high noise (variation) and low statistics. To distinguish between the two different situations explained above, and to avoid spurious halts of the region growing, then the two conditions shown in (9) have to be fulfilled to halt the region growing process:

$$
\left\{\begin{array}{l}
\max \left\{\Delta_{m 1}\right\}-\sigma\left\{\Delta_{m 1}\right\}>\max \left\{\Delta_{m 1}\right\}+\sigma\left\{\Delta_{m 1 a c c}\right\} \\
\max \left\{\Delta_{m 2}\right\}-\sigma\left\{\Delta_{m 2}\right\}>\max \left\{\Delta_{m 2 a c c}\right\}+\sigma\left\{\Delta_{m 2 a c c}\right\}
\end{array}\right.
$$

where $\Delta_{m 1 a c c}$ is a vector whose components represent the $\Delta_{m 1}(7)$ accumulated values used each time $k$ is incremented, and $\Delta_{m 2 a c c}$ is similarly defined for $\Delta_{m 2}$ (8). A real intensity histogram example is shown in Figure 4 with its corresponding mode (blue plot), $\Delta_{m 2 a c c}$ (red plot) and $\Delta_{m 2}$ (green plot) evolution, shown in Figure 5. The red arrows indicate every time the parameter $k$ is increased. As mentioned previously index $t$ iterates for an initial $k$ value $\left(k_{\text {init }}\right)$. When no more pixels are appended for that $k$ value, this is increased by $k=k+1$. After $n$ iterations ( $\mathrm{k}+\mathrm{n}$ in Figure 5$)$ a significant change of $\Delta_{m 2}$ is detected, resulting in a halt of the region grower.

It can be observed how the mode flickers at the beginning when the histogram has low statistics, and then it stabilizes until a large change is detected. This initial flickering might be discarded by checking whether there actually exists a valley between the distributions in the intensity histogram.

\subsubsection{Termination criterion based on difference between region sizes}

After several iteration with the same $k$ value, when no further pixels are appended to the region, then parameter $k$ is updated. The region grower then updates $k$ resulting in a more relaxed condition (3). Then the difference in region size is obtained as shown in (10).

$$
I_{k}=A_{k}-A_{k-1}
$$

where $A_{k}$ is the region size at iteration $k$ and $A_{k-1}$ is the region size at iteration $k-1$. The region grower considers that region being segmented is leaking in to another region if $I_{k}>5 I_{k-1}$. 


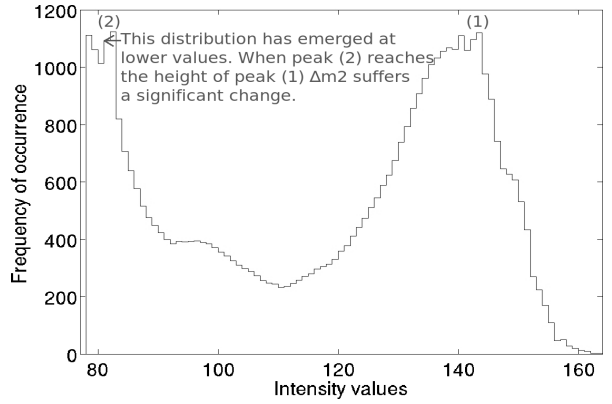

Figure 4. Example of intensity histogram where $\Delta_{m 2}$ suffers a significant change.

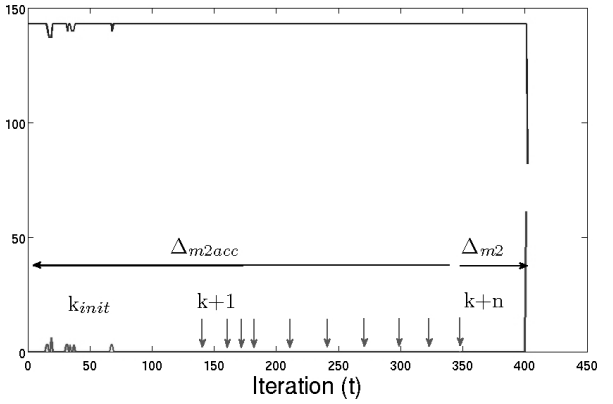

Figure 5. Evolution of the histogram mode (blue), $\Delta_{m 2 a c c}$ (red) and $\Delta_{m 2}$ (green) of intensity histogram shown in Figure 4 . The increments of the parameter $k$ are marked with red arrows.

\subsubsection{Termination criterion based on edge detection}

Before the region grower is applied to the autoradiogram, $G(i, j)$, the gradient of the image, is first computed as described in (11):

$$
G(i, j)=\sqrt{\nabla_{h} I(i, j)^{2}+\nabla_{v} I(i, j)^{2}+\nabla_{d 1} I(i, j)^{2}+\nabla_{d 2} I(i, j)^{2}}
$$

where the terms $\nabla I(i, j)$ are the finite differences of pixel $(i, j)$ with its 8 neighbours.

Having computed the gradient $G(i, j)$, every time the parameter $k$ is updated, the binary contour of the region being segmented, defined by $T(i, j)^{k}$ (membership function), is extracted, and the gradient of only those pixels belonging to the contour are summed up and normalized, dividing by the cardinality of the contour $\left(\sum T(i, j)^{k}\right)$, resulting in an updated gradient scalar measure defined by $T(i, j)^{\prime k}(12)$ :

$$
T(i, j)^{\prime k}=\frac{\sum G(i, j) T(i, j)^{k}}{\sum T(i, j)^{k}}
$$

If $T(i, j)^{\prime k}<T(i, j)^{\prime k-1}$ the region grower considers that it has missed an edge in the previous iteration $k-1$, therefore the region grower halts and considers the region segmented at iteration $k-1$ as valid.

In order to avoid premature halting of the region grower, a second condition has to be fulfilled by $T(i, j)^{\prime k-1}$ to stop the region grower. As has been mentioned, the level of statistical noise in the typical autoradiograms used here is significant, so there may be some iterations where $T(i, j)^{\prime k}$ is slightly lower than $T(i, j)^{\prime k-1}$, enough to stop the region grower, but it does not actually corresponds with a functional region. This is why a second condition to be fulfilled by $T(i, j)^{\prime k-1}$ has been introduced: $T(i, j)^{\prime k-1}$ has to be above a certain threshold to actually be considered. This threshold is $\sigma_{g}+m_{g}$, where $\sigma_{g}$ and $m_{g}$ are the standard deviation and the mode of the gradient image $G(i, j)$ respectively (considering in $G(i, j)$ only those pixels above zero).

In order to segment the rest of the objects in the image, consecutive seed pixels are located using the criterion of the next highest pixel value in the image excluding those already segmented. This pixel needs to be a minimum distance of $M$ pixels (a value of 30 was used here) from the previously segmented regions to avoid over-segmentation by growing regions too close.

The whole process is presented with images in Figure 6 using a ${ }^{3} \mathrm{H}$ labelled tissue section as example. Figure 6(a) shows the original image after pre-processing (thresholding and filtering). Figure 6(b) shows the original autoradiogram with the most important functional regions (high concentration of D1 neuroreceptors) delineated. Figure 6(c) shows the original autoradiogram after removing those regions already segmented in the previous step, and with the intensity inverted, where regions with low level uptake are represented by high intensity values. Figure 6(d) shows the original autoradiogram with the functional regions with higher level of radioligand uptake delineated (Figure 6(b)), and also the regions with lower level of uptake. 


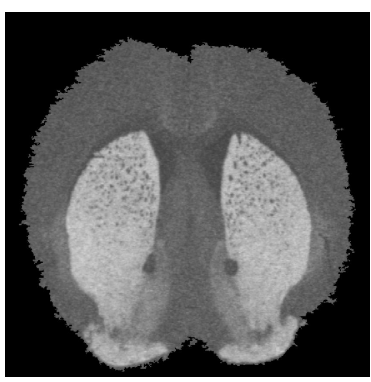

(a)

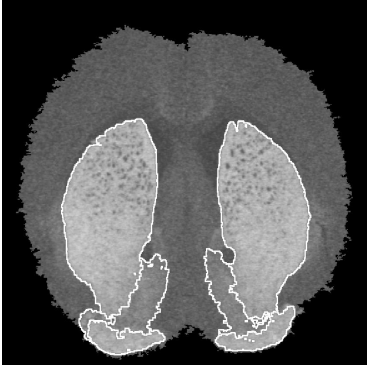

(b)

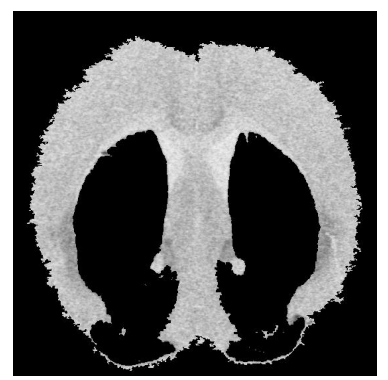

(c)

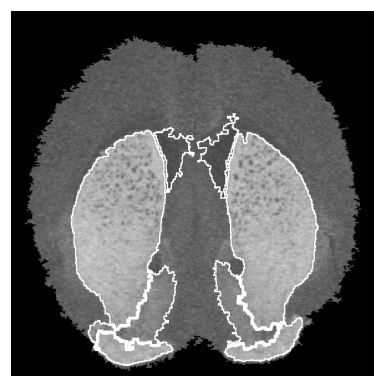

(d)

Figure 6. In this Figure the segmentation process is summarized. (a) represents the original image, a ${ }^{3}[\mathrm{H}] \mathrm{SCH}-23390$ labelled tissue section, (b) represents the segmentation of the key structures with higher binding level, (c) represents the intensity inverted residual image with the previously segmented structures removed and (d) represents the final tissue section with the key structures with lower and higher binding levels segmented.

\section{VALIDATION}

The performance of the segmentation algorithm is assessed measuring how often the algorithm results in a correct decision, in order to predict how this will perform when used routinely. The issue here is that pixel counting regions as correct or incorrectly segmented regions is too complex due to lack of absolute ground truth in a functional biological image. We have used therefore a set of simple test simulated images where the ground-truth and noise are known. The metrics used to evaluate the segmentation technique presented here is based on measuring the percentage of over-segmented and under-segmented pixels depending on the noise added to the simulated image. ${ }^{15}$

The ideal simulated image, shown in Figure 7(a), has been deliberately corrupted adding Gaussian noise with different values of variance, representing the typical statistical noise present in these kinds of imaging applications, and by blurring the edges filtering the ideal image with a Gaussian spatial filter using different variance values. Figures 7(b) and 7(c) represent the ideal image blurred with a $\sigma=5$ pixels PSF and with additive Gaussian noise with $\mu=0$ and $\sigma=2$ and $\sigma=6$ respectively. These $\sigma$ values are typically found in autoradiographic data.

These variance values used in the blurring process correspond to the system impulse response, defined by the Point Spread Function (PSF), that are observed in the different imaging modalities. In the case of Autoradiography this blurring effect is mainly due to the $\beta$ particle mean range $\left(\sim 0.3 \mu \mathrm{m}\right.$ for ${ }^{3} \mathrm{H}, \sim 13 \mu \mathrm{m}$ for ${ }^{14} \mathrm{C}$ and ${ }^{35} \mathrm{~S}$ ) and by the deposited charge diffusion process in the active layer of a digital imaging device. Based on experimental work two different scenarios are considered here. For low energy radioisotopes, such as ${ }^{3} \mathrm{H}$, a $\sigma=2$ pixels has been used, and for medium energy radioisotopes such as ${ }^{14} \mathrm{C}$ and ${ }^{35} \mathrm{~S}$, a $\sigma=5$ pixels has been used.

The assessment of this image segmentation algorithm is based on the CNR (13) of the image. Mean intensity values and noise values have been directly measured in a variety of autoradiograms labelled with different radioisotopes to use real values in the assessment of the algorithm. The measure of the assessment is based on the percentage of over-segmented and under-segmented pixels of each tissue for different CNR values.

$$
C N R=\frac{S_{A B}}{\sigma_{b c k}}
$$

where $S_{A B}$ is the difference $\mu_{A^{-}} \mu_{B}$, being $\mu_{A}$ and $\mu_{B}$ the mean intensities of ROIs in tissues A and B respectively, and $\sigma_{b c k}$ is the standard deviation of the background, considered as the noise. The Gaussian noise 
added to the test image has the same variance for the three structures (tissue A, tissue B and background), therefore the standard deviation measured in any of the regions is the same.

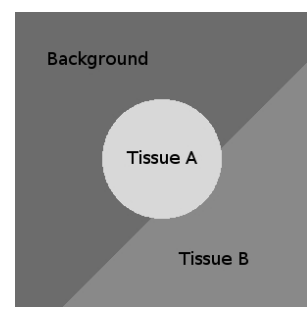

(a)

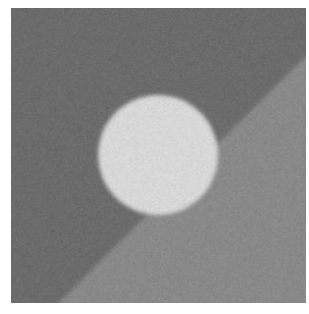

(b)

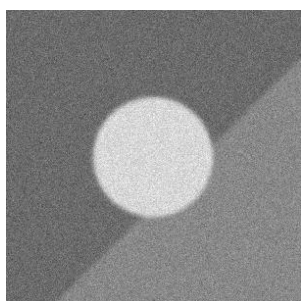

(c)

Figure 7. Simulated noiseless image (a), simulated image blurred with a $\sigma=5$ PSF and additive Gaussian noise ( $\mu=0$ and $\sigma=2$ ) (b)and simulated image blurred with a $\sigma=5$ PSF and additive Gaussian noise $(\mu=0$ and $\sigma=6)(\mathrm{c})$.

It is observed from (13) that the CNR depends on the difference of intensities between the two tissues under study and the statistical noise present. In the approach used in this work the difference of intensities between tissues is fixed to a value measured directly from autoradiographic data, but the statistical noise is increased. After measuring manually the intensity in some of our autoradiograms the values used for the assessment here are $S_{A B}=40$ (tissues A and B) and $S_{B b c k}=35$ (tissues B and background). The standard deviation measured in different regions of several autoradiograms varied from 8 down to 3 in some cases. In this work a range of 0.5 up to 10 has been analyzed, resulting in a CNR range of $81.0(\sigma=0.5)$ down to 4.0 $(\sigma=10)$, as observed in Figure 8. In Figure 9 the percentage of over-segmented and under-segmented pixels are shown when segmenting tissue A and tissue B. Each combination (PSF and $\sigma$ ) has been repeated over 30 times with different statistical noise, therefore a statistical mean and standard deviation for each combination has been obtained, as shown in Figure 9 with error bars. Those error bars that show significant values for Tissue B (shown in black in Figure 9(a) and in red in Figure 9(b)) represent situations where either the region was barely segmented due to the presence of high statistical noise (Figure 9(a)), or where the segmentation completely leaked in the background tissue.

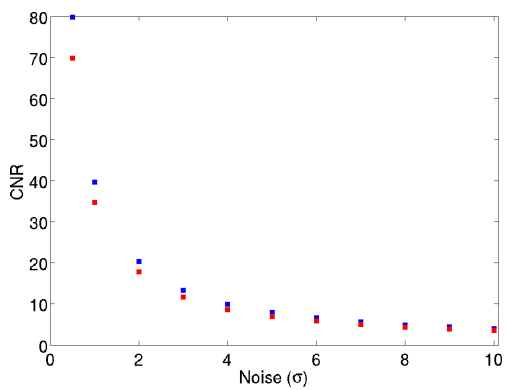

Figure 8. CNR measured between Tissue $\mathrm{A}$ and $\mathrm{B}$ (blue) and $\mathrm{B}$ and background (red) with statistical noise $\sigma$.

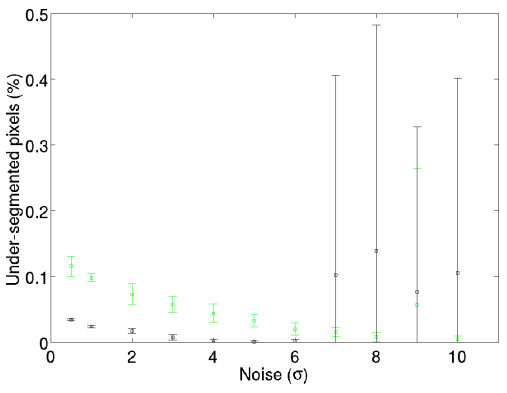

(a)

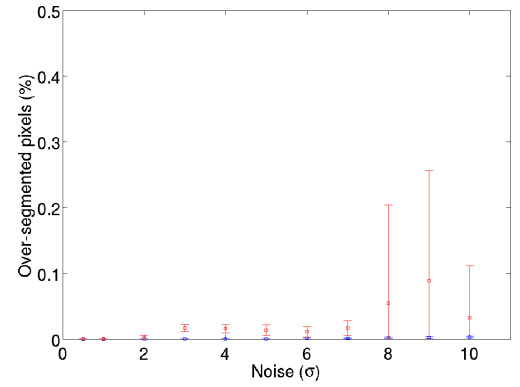

(b)

Figure 9. Percentage of under-segmented (left) and over-segmented (right) pixels of segmentation of tissue A (green and blue) and tissue B (black and red).

In Figure 9(a) it is observed how Tissue A slightly suffers under-segmentation $(<15 \%)$ for low values of $\sigma$ resulting from the blurring effect in the edges. As the noise increases the blurring effect takes less importance, therefore the number of under-segmented pixels decreases. Tissue B suffers in some cases of under-segmentation for high values of noise $(\sigma>6)$ due to premature halts. In Figure 9(b) Tissue A never 
suffers of over-segmentation, while Tissue B suffers of over-segmentation in some cases when $\sigma>7$. In general this demonstrates how this approach is able to segment reliably data with noise $\sigma<7$, corresponding to a $\mathrm{CNR} \simeq 6$.

\section{RESULTS}

Several pre-clinical images have been used to assess the performance of this segmentation algorithm. Only a few examples are presented in this work.

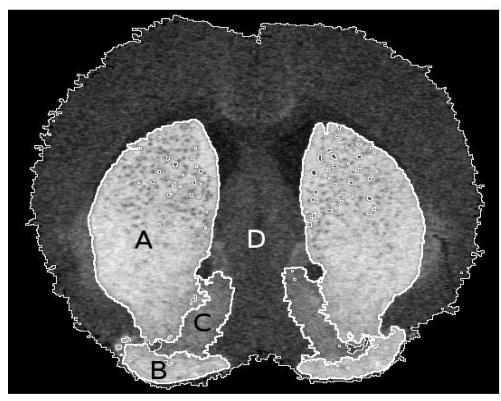

Figure 10. Segmented autoradiogram labelled with ${ }^{3} \mathrm{H}$

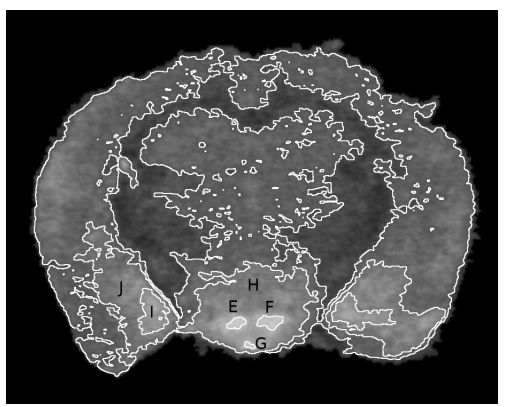

Figure 11. Segmented autoradiogram labelled with ${ }^{35} \mathrm{~S}$.

The CNR between different key anatomical regions, denoted by letters, in the sections shown in Figure 10 and Figure 11 has been measured. For comparison, the CNR in CT and MR data is typically of the order of 200, 300 (3T scanner) and 100 (1.5T scanner) respectively.

It can be seen that key areas of high and low uptake in these images have been successfully segmented. This can speed up the analysis by allowing corresponding areas of anatomy be anatomically labelled. The CNR values measured between the segmented regions are shown in Table 1 for Figure 10, and Table 2 for Figure 11.

Table 1. CNR values for the regions shown in Figure 10

\begin{tabular}{llllllll}
\hline$C N R_{A-C}$ & 4.4 & $C N R_{A-D}$ & 10.3 & $C N R_{B-C}$ & 4.5 & $C N R_{C-D}$ & 4.4 \\
Table 2. CNR values for the regions shown in Figure 11 & \\
\hline$C N R_{E-H}$ & 5.7 & $C N R_{F-H}$ & 4.6 & $C N R_{G-H}$ & 3.5 & $C N R_{I-J}$ & 2.3
\end{tabular}

\section{CONCLUSIONS}

A segmentation approach, based on region growing, has been presented for segmenting poor contrast to noise ratio images. Due to the typical high levels of statistical noise present in this kind of functional data, certain complexity is added to the typical similarity criterion used in region growing. The termination criteria have also been an important part of this work as premature halts or growing on different tissues are very likely to occur when high levels of noise are present. The termination criteria detailed here demonstrate to be able to avoid these two situations in synthetic images with high levels of statistical noise, and in real autoradiographic data.

Initial results presented here show that this technique is able to segment areas of a variety of different regions seen in autoradiographic data. This technique could potentially be used for other functional pre clinical imaging modalities such as PET, SPECT and fMRI.

The validation of this approach has been presented by modifying the statistical noise of the image, demonstrating how the algorithm is able to reliably segment images with noise up to $\sigma=7$, corresponding to a $\mathrm{CNR} \simeq 6$. Results obtained from autoradiographic data demonstrate how this limit can be pushed even further in practical cases. 


\section{Acknowledgment}

This work is supported by the RC-UK Basic Technology Multidimensional Integrated Intelligent Imaging $\left(\mathrm{MI}^{3}\right)$ programme $(\mathrm{GR} / \mathrm{S} 85733 / 01)$.

\section{REFERENCES}

[1] Pal, N. R. and Pal, S. K., "A review on image segmentation techniques," Pattern Recognition 26, 12771294 (1993).

[2] Hatt, M., Lamare, F., Boussion, N., Turzo, A., Collet, C., Salzenstein, F., Roux, C., Jarritt, P., Carson, K., Rest, C. C.-L., and Visvikis, D., "Fuzzy hidden markov chains segmentation for volume determination and quantitation in PET," Physics in Medicine and Biology 52(12), 3467-3491 (2007).

[3] Saad, A., Smith, B., Hamarneh, G., and Moller, T., "Simultaneous segmentation, kinetic parameter estimation, and uncertainty visualization of dynamic PET images," Med Image Comput Comput Assist Interv Int Conf Med Image Comput Comput Assist Interv 10(2), 726-733 (2007).

[4] Kim, J., Cai, W., Feng, D., and Eberl, S., "Segmentation of VOI from multidimensional dynamic PET images by integrating spatial and temporal features," IEEE Transactions on Information Technology in Biomedicine 10, 637-646 (2006).

[5] Chen, J. L., Gunn, S. R., Nixon, M. S., and Gunn, R. N., "Markov random field models for segmentation of PET images," in [IPMI '01: Proceedings of the 17th International Conference on Information Processing in Medical Imaging], 468-474, Springer-Verlag, London, UK (2001).

[6] Zhu, W. and Jiang, T., "Automation segmentation of PET image for brain tumors," Nuclear Science Symposium Conference Record, 2003 IEEE 4, 2627-2629 Vol.4 (19-25 Oct. 2003).

[7] Hojjatoleslami, S. A. and Kittler, J., "Region growing: A new approach," IEEE Transactions on Image Processing 7, 1079-1084 (1998).

[8] Pohle, R. and Toennies, K. D., "Segmentation of medical images using adaptive region growing," Proc. SPIE Medical Imaging 4322, 1337-46 (2001).

[9] Yi, J. and Ra, J. B., "A locally adaptive region growing algorithm for vascular segmentation," International Journal of Imaging Systems and Technology 13, 208-214 (2003).

[10] Lu, Y., Jiang, T., and Zang, Y., "Region growing method for the analysis of functional MRI data," Neuroimage 20, 455-465 (2003).

[11] Mancas, M., Gosselin, B., and Macq, B., "Segmentation using a region-growing thresholding," in [Image Processing: Algorithms and Systems IV. Edited by Dougherty, Edward R.; Astola, Jaakko T.; Egiazarian, Karen O. Proceedings of the SPIE, Volume 5672, pp. 388-398 (2005).], Dougherty, E. R., Astola, J. T., and Egiazarian, K. O., eds., Presented at the Society of Photo-Optical Instrumentation Engineers (SPIE) Conference 5672, 388-398 (Mar 2005).

[12] Lee, Y. J., Park, J. M., and Park, H. W., "Mammographic mass detection by adaptive thresholding and region growing," International Journal of Imaging Systems and Technology 11, 340-346 (2001).

[13] Pan, Z. and Lu, J., "A Bayes-Based Region-Growing algorithm for medical image segmentation," Computing in Science and Engineering 9, 32-38 (2007).

[14] Perona, P. and Malik, J., "Scale-Space and Edge Detection Using Anisotropic Diffusion," IEEE Trans. on Pattern Analysis and Machine Intelligence 12(7), 629-39 (1990).

[15] Bowyer, K., "Validation of medical image analysis techniques," in [Handbook of Medical Imaging, Vol. 2: Medical Image Processing and Analysis], New York: SPIE (2000). 